\title{
Laboratory detection of the rotational-tunnelling spectrum of the hydroxymethyl radical, $\mathrm{CH}_{2} \mathrm{OH}$
}

\author{
C. Bermudez ${ }^{1}$, S. Bailleux ${ }^{1}$, and J. Cernicharo ${ }^{2}$ \\ ${ }^{1}$ Univ. Lille, CNRS, UMR 8523 - PhLAM - Physique des Lasers, Atomes et Molécules, 59000 Lille, France \\ e-mail: stephane.bailleux@univ-lille1.fr \\ 2 Instituto de Ciencia de Materiales de Madrid, CSIC, Group of Molecular Astrophysics, C/Sor Juana Inés de la Cruz 3, \\ 28049 Cantoblanco, Madrid, Spain
}

Received 9 August 2016 / Accepted 30 October 2016

\begin{abstract}
Context. Of the two structural isomers of $\mathrm{CH}_{3} \mathrm{O}$, methoxy is the only radical whose astronomical detection has been reported through the observation of several rotational lines at 2 and $3 \mathrm{~mm}$ wavelengths. Although the hydroxymethyl radical, $\mathrm{CH}_{2} \mathrm{OH}$, is $\mathrm{known}$ to be thermodynamically the most stable (by $\sim 3300 \mathrm{~cm}^{-1}$ ), it has so far eluded rotational spectroscopy presumably because of its high chemical reactivity.

Aims. Recent high-resolution ( $10 \mathrm{MHz}$ ) sub-Doppler rovibrationally resolved infrared spectra of $\mathrm{CH}_{2} \mathrm{OH}$ (symmetric $\mathrm{CH}$ stretching $a$-type band) provided accurate ground vibrational state rotational constants, thus reviving the quest for its millimeter-wave spectrum in laboratory and subsequently in space.

Methods. The search and assignment of the rotational spectrum of this fundamental species were guided by our quantum chemical calculations and by using rotational constants derived from high-resolution IR data. The hydroxymethyl radical was produced by hydrogen abstraction from methanol by atomic chlorine.

Results. Ninety-six $b$-type rotational transitions between the $v=0$ and $v=1$ tunnelling sublevels involving 25 fine-structure components of $Q$ branches (with $K_{a}=1 \leftarrow 0$ ) and 4 fine-structure components of $R$ branches (assigned to $K_{a}=0 \leftarrow 1$ ) were measured below $402 \mathrm{GHz}$. Hyperfine structure alternations due to the two identical methylenic hydrogens were observed and analysed based on the symmetry and parity of the rotational levels. A global fit including infrared and millimeter-wave lines has been conducted using Pickett's reduced axis system Hamiltonian. The recorded transitions (odd $\Delta K_{a}$ ) did not allow us to evaluate the Coriolis tunnelling interaction term. The comparison of the experimentally determined constants for both tunnelling levels with their computed values secures the long-awaited first detection of the rotational-tunnelling spectrum of this radical. In particular, a tunnelling rate of $139.73 \pm 0.10 \mathrm{MHz}\left(4.6609(32) \times 10^{-3} \mathrm{~cm}^{-1}\right)$ was obtained along with the rotational constants, electron spin-rotation interaction parameters and several hyperfine coupling terms.

Conclusions. The laboratory characterization of $\mathrm{CH}_{2} \mathrm{OH}$ by millimeter-wave spectroscopy now offers the possibility for its astronomical detection for the first time.
\end{abstract}

Key words. molecular data - ISM: molecules - submillimeter: ISM

\section{Introduction}

The hydroxymethyl radical, $\mathrm{CH}_{2} \mathrm{OH}$, is involved as an intermediate species in many chemical reaction systems, and its study spans a wide range of fields, such as in tropospheric chemistry (Atkinson 1990; Pagsberg et al. 1989; Nesbitt et al. 1989) and hydrocarbon (e.g. methane and methanol) oxidation processes (Lin et al. 1999, 2000; Rissanen et al. 2013; Rasmussen et al. 2008). It is also supposed to be an abundant molecule in space because of the ubiquitous methanol molecule, where its less stable structural isomer radical, $\mathrm{CH}_{3} \mathrm{O}$, has recently been unexpectedly detected by Cernicharo et al. (2012) towards the cold dark core B1-b. In such cold dark regions a significant $\mathrm{CH}_{3} \mathrm{O} / \mathrm{CH}_{2} \mathrm{OH}$ ratio in the gas phase would be indicative of either $\mathrm{CH}_{3} \mathrm{O} / \mathrm{CH}_{2} \mathrm{OH}$ photo-desorption or their direct formation in the gas phase. Laboratory experiments have indeed revealed that $\mathrm{CH}_{2} \mathrm{OH}$ (in contrast to $\mathrm{CH}_{3} \mathrm{O}$ ) is formed by $\mathrm{UV}$ photolysis of interstellar ice analogues containing $\mathrm{CH}_{3} \mathrm{OH}$ (see e.g. Gerakines et al. 1996). Other similar experiments (see e.g. Watanabe \& Kouchi 2002; Watanabe et al. 2007) indicated that the detections of $\mathrm{H}_{2} \mathrm{CO}$ and $\mathrm{CH}_{3} \mathrm{OH}$ by UV/ion irradiation of
$\mathrm{CO}: \mathrm{H}_{2} \mathrm{O}$ ices suggest that $\mathrm{CH}_{3} \mathrm{O}$ and/or $\mathrm{CH}_{2} \mathrm{OH}$ are the intermediate species following the chain reaction $\mathrm{H}_{2} \mathrm{CO}+\mathrm{H} \rightarrow$ $\mathrm{CH}_{3} \mathrm{O} / \mathrm{CH}_{2} \mathrm{OH}+\mathrm{H} \rightarrow \mathrm{CH}_{3} \mathrm{OH}$. Therefore, taking into account the large methanol abundance in B1-b (Öberg et al. 2010), the possibility to form both methoxy and hydroxymethyl radicals has to be considered.

The high chemical reactivity of $\mathrm{CH}_{2} \mathrm{OH}$ under laboratory conditions makes it difficult to study experimentally, and its rotational spectrum has always eluded microwave spectroscopists, a fact that has so far hampered its radioastronomical discovery.

In view of its general chemical importance as an intermediate species, especially in modelling the chemistry of interstellar medium, this radical has been the subject of very many studies. An exhaustive list of bibliographic references can be found in Johnson \& Hudgens (1996) and in Roberts et al. (2013), for example. In this latter spectroscopic work, which was done in the Nesbitt Laboratory, accurate $B$ and $C$ rotational constants in the ground state were derived from the analysis of the highresolution, sub-Doppler, near-infrared (NIR) spectrum of the $v_{3}$ symmetric $\mathrm{CH}$ stretching band. 
Table 1. Structure of $\mathrm{CH}_{2} \mathrm{OH}$ optimized at $C_{\mathrm{s}}$ symmetry calculated at the B3LYP/6-311++G(d, p) level of theory.

\begin{tabular}{ccccccc}
\hline \hline $\mathrm{OH}_{1}$ & $\mathrm{CO}$ & $\mathrm{CH}_{2}$ & $\mathrm{CH}_{3}$ & $\angle \mathrm{COH}_{1}$ & $\angle \mathrm{H}_{3} \mathrm{CO}$ & $\angle \mathrm{H}_{2} \mathrm{CH}_{3}$ \\
\hline 0.962 & 1.365 & 1.077 & 1.080 & 110.02 & 120.51 & 124.43 \\
\hline
\end{tabular}

Notes. Bond lengths in $\AA$, bond angles in degree. To avoid confusion, the three protons are labelled $\mathrm{H}_{1-3}$, where $\mathrm{H}_{1}$ is the hydroxyl proton, and $\mathrm{H}_{2,3}$ the two methylenic protons. $\mathrm{H}_{1}$ and $\mathrm{H}_{3}$ are located at the same side of the $\mathrm{CO}$ chemical bond.

Our research presented in this paper, the first identification of the rotational-tunnelling spectrum of $\mathrm{CH}_{2} \mathrm{OH}$, relies for the most part on this recent fully rotationally resolved high-resolution IR spectra of the hydroxymethyl radical.

\section{Prediction of the spectrum}

\subsection{Quantum chemical calculations}

The rotational spectrum of $\mathrm{CH}_{2} \mathrm{OH}$ was predicted on the basis of ab initio geometrical structure optimization to provide the molecular parameters that are needed to assist with the search for and identification of this spectrum. These are the rotational and quartic centrifugal distortion constants, the electron spin-rotation interaction tensor that is due to the unpaired electron, and the isotropic (Fermi contact) and anisotropic contributions (magnetic dipole-dipole interaction) of the three protons that account for the hyperfine structures. At equilibrium, $\mathrm{CH}_{2} \mathrm{OH}$ is a non-planar species belonging to the $C_{1}$ point group with unequal $\mathrm{CH}$ bond lengths. However, it is well accepted (Johnson \& Hudgens 1996; Kamarchik et al. 2012) that this radical in its ground vibrational state effectively behaves as a planar molecule of $C_{\mathrm{s}}$ symmetry with a ${ }^{2} \mathrm{~A}^{\prime \prime}$ electronic ground state. Therefore the $C_{\mathrm{s}}$ symmetry-constrained geometry optimization has been performed by employing the B3LYP/6-311++G(d, p) method. The Gaussian package (Frisch et al. 2009) has been used, and the resulting geometry, in close agreement with that of Kamarchik et al. (2012), is cast in Table 1.

The computed components of the electric dipole moment are $-0.13 \mathrm{D}$ and $+1.64 \mathrm{D}$ for $\mu_{a}$ and $\mu_{b}$, respectively. The molecular parameters derived from the structure are given in Tables 2-4. Roberts et al. (2013) obtained from high-resolution $(\sim 10 \mathrm{MHz})$ sub-Doppler rovibrationally resolved IR spectra the $B$ and $C$ rotational constants in the ground vibrational state (29832(18) and 25 950(15) MHz, respectively), and our quantum chemically computed values compare well (Table 2 ). The asymmetry parameter $K=-0.95$ inferred from the rotational constants shows that $\mathrm{CH}_{2} \mathrm{OH}$ is a near-prolate symmetric rotor with an electric dipole moment that is mainly imposed by the $\mathrm{OH}$ bond.

\subsection{Tunnelling splitting and nuclear spin statistics}

At $C_{\mathrm{s}}$ conformation, $\mathrm{CH}_{2} \mathrm{OH}$ has a double minimum potential with a finite torsional barrier height $\left(\sim 1650 \mathrm{~cm}^{-1}\right.$, Johnson \& Hudgens 1996), allowing tunnelling of the hydroxyl proton between two isoenergetic mirror frameworks. The tunnelling doublings were clearly brought to light in the highresolution NIR spectra of $v_{1}(\mathrm{OH}$ stretching, Wang et al. 2014a), $v_{2}$ (CH asymmetric stretching, Chang et al. 2014), and $v_{3}(\mathrm{CH}$ symmetric stretching, Wang et al. 2014b) bands measured in the Nesbitt laboratory. An upper limit of $10^{-3} \mathrm{~cm}^{-1}(30 \mathrm{MHz})$ for the tunnelling rate was also estimated for the ground vibrational
Table 2. Energy separation between tunnelling substates $\Delta E_{01}$, rotational $(\mathrm{MHz})$ and quartic centrifugal distortion constants $(\mathrm{kHz})$ of $\mathrm{CH}_{2} \mathrm{OH}$ in the $A$ reduction, $\mathrm{I}^{\mathrm{r}}$ representation.

\begin{tabular}{|c|c|c|}
\hline \multirow[t]{2}{*}{ Parameter } & \multirow[t]{2}{*}{$\mathrm{Ab}$ initio $^{a}$} & Experiment $^{b}$ \\
\hline & & $v=0 \quad v=1$ \\
\hline$\Delta E_{01}$ & & $139.73(10)$ \\
\hline$A$ & 195902.0 & $194540.09(20)$ \\
\hline$B$ & 29910.9 & $29844.038(38) \quad 29844.188(38)^{c}$ \\
\hline$C$ & 25948.9 & $25946.463(17) \quad 25946.187(17)^{c}$ \\
\hline$\Delta_{N}$ & 63.1 & $68.48(12)$ \\
\hline$\Delta_{N K}$ & 566.5 & $760(13)$ \\
\hline$\Delta_{K}$ & 5326.5 & $5326.5^{d}$ \\
\hline$\delta_{N}$ & 8.5 & $8.5^{d}$ \\
\hline$\delta_{K}$ & 421.6 & $383(13)$ \\
\hline
\end{tabular}

Notes. ${ }^{(a)}$ Rotational and quartic centrifugal distortion constants calculated at the B3LYP/6-311++G(d, p) level of theory using $C_{\mathrm{s}}$ symmetryconstrained geometry optimization. ${ }^{(b)}$ Ground vibrational state rotational and quartic centrifugal distortion constants. Except for $B$ and $C$, identical parameters have been used for both tunnelling levels $(v=0,1)$ in the least-squares analysis. $1 \sigma$ error given in parentheses apply to the last digits. ${ }^{(c)}$ Derived value. The $B(v=1)-B(v=0)$ and $C(v=1)-C(v=0)$ were fitted instead to avoid the correlation between the rotational constants of the two tunnelling levels. The least-squares analysis gave for these differences $+0.150(6)$ and $-0.276(5) \mathrm{MHz}$, respectively. ${ }^{(d)}$ Fixed in the least-squares analysis at the computed value.

Table 3. Electron spin-rotation $(\mathrm{MHz})$ and their quartic centrifugal distortion coupling constants $(\mathrm{MHz})$ of $\mathrm{CH}_{2} \mathrm{OH}$ in the $A$ reduction, $\mathrm{I}^{\mathrm{r}}$ representation.

\begin{tabular}{ccc}
\hline \hline Parameter & Ab initio $^{a}$ & \multicolumn{1}{c}{ Experiment $^{b}$} \\
\hline$\epsilon_{a a}$ & -506.5 & \multicolumn{1}{c}{$-517.5(18)$} \\
$\epsilon_{b b}$ & -121.4 & $-124.97(36)$ \\
$\epsilon_{c c}$ & 9.3 & $-3.18(42)$ \\
$\Delta_{N}^{S}$ & & $0.0312(21)$ \\
$\Delta_{N K}^{S}+\Delta_{K N}^{S}$ & & $1.226(74)$ \\
$\Delta_{K}^{S}$ & & $0.0^{c}$ \\
\hline
\end{tabular}

Notes. The two off-diagonal coupling constants $\left(\epsilon_{a b}+\epsilon_{b a}\right) / 2$ $(-85.1 \mathrm{MHz})$ and $\left(\epsilon_{a b}-\epsilon_{b a}\right) / 2(41.9 \mathrm{MHz})$ have been omitted in the fit. (a) Electron spin-rotational tensor computed employing the B3LYP/6$311++\mathrm{G}(\mathrm{d}, \mathrm{p})$ level of theory at $C_{\mathrm{s}}$ conformation. ${ }^{(b)}$ The $1 \sigma$ error given in parentheses applies to the last digits. The same constants have been used for fitting transitions arising from the two ground vibrational state tunnelling levels. ${ }^{(c)}$ Fixed parameter.

state using high-level $(\mathrm{CCSD}(\mathrm{T}) / \mathrm{V} n \mathrm{Z}-\mathrm{F} 12$ with $n=2,3) \mathrm{ab}$ initio computations (Nesbitt et al. 2014). To clarify the allowed electric dipole rotational-tunnelling transitions in the ground vibrational state, the two tunnelling sublevels of the rigid rotor levels of $\mathrm{CH}_{2} \mathrm{OH}\left(C_{\mathrm{s}}\right.$ symmetry) can be labelled with the symmetry species of the $C_{2 \mathrm{v}}(\mathrm{M})$ molecular symmetry group. This group is isomorphic with the permutation-inversion symmetry group $\left(G_{4}\right)$ of this non-rigid radical (Bunker \& Jensen 1998). Fermi-Dirac statistics dictates that the complete internal wavefunction $\Phi_{\text {tot }}=\Phi_{\text {elec }} \Phi_{\text {rot }} \Phi_{\text {tun }} \Phi_{\text {vib }} \Phi_{\text {ns }}$ must change in sign under the sole exchange of the two identical methylenic hydrogen atoms, corresponding to the $C_{2 a}$ operation. Therefore the symmetry of $\Phi_{\text {tot }}$ is $B_{1}$ or $B_{2}$. The electronic wavefunction $\Phi_{\text {elec }}$ is of $\mathrm{B}_{1}$ symmetry since the $C_{2 \mathrm{v}}$ radical has an $\mathrm{X}^{2} \mathrm{~B}_{1}$ ground state 
Table 4. Isotropic (Fermi-contact terms $\left.a_{F}\right)$ and anisotropic $\left(T_{i j}\right)$ hyperfine interaction constants $(\mathrm{MHz})$ of the three protons of $\mathrm{CH}_{2} \mathrm{OH}$.

\begin{tabular}{lcc}
\hline \hline Parameter & Ab initio $^{a}$ & Experiment $^{b}$ \\
\hline Hydroxyl proton $\left(\mathrm{H}_{1}\right)$ & & \\
$\quad a_{F}$ & -12.4 & $-12.4^{c}$ \\
$3 / 2 T_{a a}$ & -2.85 & $-2.85^{c}$ \\
$1 / 4\left(T_{b b}-T_{c c}\right)$ & 5.1 & $4.268(28)$ \\
Methylenic protons $\left(\mathrm{H}_{2}, \mathrm{H}_{3}\right)^{d}$ & & \\
$a_{F}$ & -63.1 & $-52.19(86)$ \\
$3 / 2 T_{a a}$ & -33.4 & $-36.7(28)$ \\
$1 / 4\left(T_{b b}-T_{c c}\right)$ & 5.5 & $3.904(27)$ \\
\hline
\end{tabular}

Notes. (a) Hyperfine interaction terms obtained at the B3LYP/6$322++G(d, p)$ level of theory. The calculated off-diagonal coupling constants of the magnetic dipole-dipole tensors have been omitted in the fit: $T_{a b}\left(\mathrm{H}_{1}\right)=17.8 \mathrm{MHz}, T_{a b}\left(\mathrm{H}_{2}, \mathrm{H}_{3}\right)=30.7 \mathrm{MHz} .{ }^{(b)}$ The $1 \sigma$ error given in parentheses applies to the last digits. The same constants have been used to fit transitions from both tunnelling levels. ${ }^{(c)}$ Fixed at the computed value in the least-squares analysis. ${ }^{(d)}$ The tunnelling motion implies the equivalence of these two protons.

Table 5. Symmetry species and statistical weights of the complete internal wavefunction for the lower $(v=0)$ and upper $(v=1)$ tunnelling sublevels for each $N_{K_{a} K_{c}}$ level.

\begin{tabular}{cccccc}
\hline \hline$K_{a} K_{c}$ & \multirow{2}{*}{$\Phi_{\text {rot }}$} & \multicolumn{2}{c}{$\Phi_{\text {tot }}$} & \multicolumn{2}{c}{ Statistical Weights } \\
& & $v=0$ & $v=1$ & $v=0$ & $v=1$ \\
\hline e e & $A_{1}$ & $B_{1}$ & $B_{2}$ & 3 & 1 \\
e o & $A_{2}$ & $B_{2}$ & $B_{1}$ & 3 & 1 \\
o e & $B_{2}$ & $B_{2}$ & $B_{1}$ & 1 & 3 \\
o o & $B_{1}$ & $B_{1}$ & $B_{2}$ & 1 & 3 \\
\hline
\end{tabular}

Bruna \& Grein (1998; the subscript 1 means symmetric with respect to the $\sigma_{\mathrm{v}}(a b)$ operator). The ground vibrational state being always symmetric, $\Phi_{\text {vib }}$ is $\mathrm{A}_{1}$. Regarding the tunnelling wavefunction $\Phi_{\text {tun }}$, states of $\mathrm{A}^{\prime}$ symmetry in the rigid radical correlate with those of $A_{1}$ and $B_{2}$ symmetry when tunnelling is feasible, while $\mathrm{A}^{\prime \prime}$ states separate into $\mathrm{A}_{2}$ and $\mathrm{B}_{1}$ states. Thus $\Phi_{\text {tun }}$ belongs to $\mathrm{A}_{1}$ for the lower $(v=0)$ and $\mathrm{B}_{2}$ for the higher $(v=1)$ tunnelling components. Coupling the two identical methylenic proton spin angular momenta to yield $I_{\mathrm{H}}$, nuclear spin states having $I_{\mathrm{H}}=1$ produce three states of $\mathrm{A}_{1}$ symmetry, and those having $I_{\mathrm{H}}=0$ give rise to one state of symmetry $\mathrm{B}_{1}$. Then the total nuclear spin wavefunction $\Phi_{\mathrm{ns}}$ generates the representation $3 \mathrm{~A}_{1} \oplus \mathrm{B}_{1}$. Finally, the rotational wavefunctions $\Phi_{\text {rot }}$ are $\mathrm{A}_{1}$ or $\mathrm{A}_{2}$ if $K_{a}$ is even and $\mathrm{B}_{1}$ or $\mathrm{B}_{2}$ for odd $K_{a}$. The symmetry labelling of the complete internal wavefunction obtained for each $K_{a} K_{c}$ parity is summarized in Table 5, where the nuclear spin weights (in the case of unresolved hyperfine structure) are also indicated. The $v_{3}$ (symmetric $\mathrm{CH}$ stretch) vibrationally excited state being also of $A_{1}$ symmetry, the selection rules for the rovibrationaltunnelling transitions (inserted in the global fit) are identical to those in the ground vibrational state.

Figure 1 depicts the energy level diagram of the $\mathrm{CH}_{2} \mathrm{OH}$ radical. The sublevels of $\mathrm{B}_{1}$ symmetry have the lowest tunnelling energy $(v=0)$ when $K_{a}+K_{c}$ is even, with a statistical weight of 3 (1) if $K_{a}$ is even (odd). The situation is reversed for odd $K_{a}+K_{c}$, that is, these sublevels correspond to those with the highest tunnelling energy $(v=1)$, with a statistical weight of 1 (3) if $K_{a}$ is even (odd).

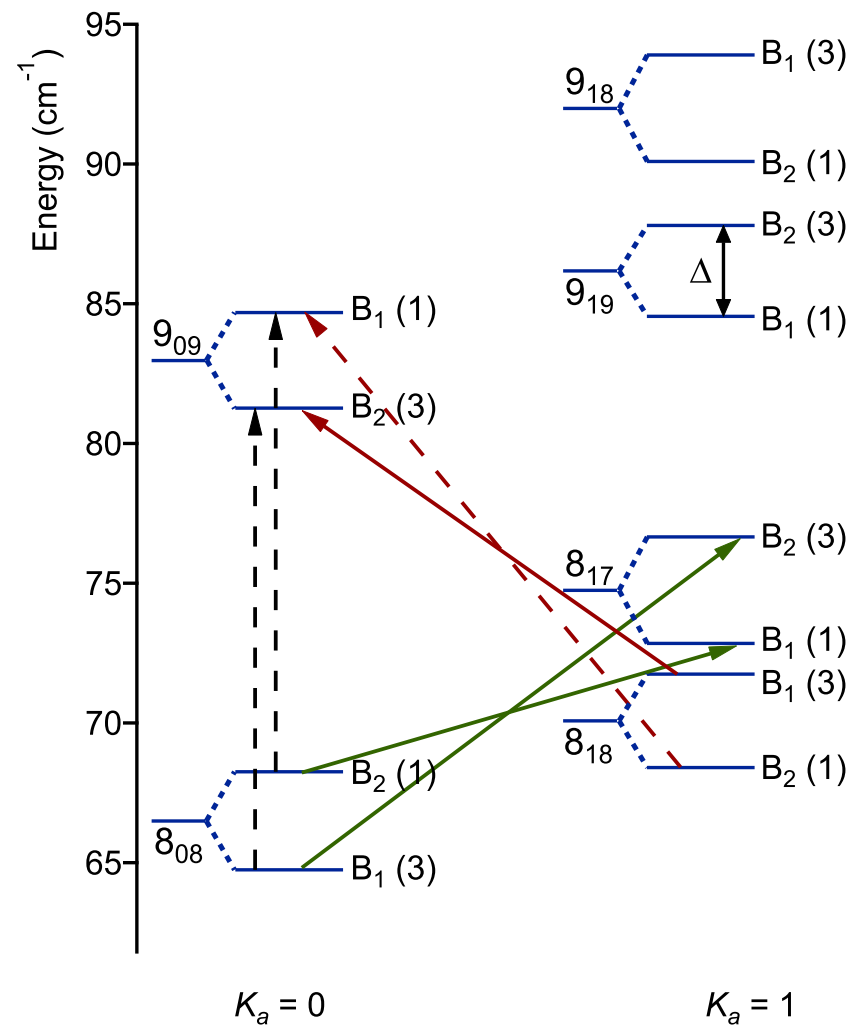

Fig. 1. Energy level diagram showing the doubling of the rigid rotor levels (identified by $N_{K_{a} K_{c}}$ ) for $K_{a}=0,1$ into tunnelling sublevels labelled with the symmetry species of the complete internal wavefunction of $\mathrm{CH}_{2} \mathrm{OH}$ in the $\left.\mathrm{C}_{2 \mathrm{v}} \mathrm{(M}\right)$ group. The statistical weights arising from the permutation of the two identical methylenic hydrogen nuclei are indicated between parentheses. Levels with a weight of 3 correspond to ortho $-\mathrm{CH}_{2} \mathrm{OH}$ and those with a weight of 1 correspond to the para isomer of the radical. Examples of $a$-type transitions (not observed) are depicted as black, dashed lines, while representatives of $b$-type (observed) $Q$ - and $R$-branch transitions are displayed in green and red, respectively. For clarity the tunnelling splittings $(\Delta)$ are 800 times the actual ones, and the fine- and hyperfine-structure splittings are not illustrated.

The allowed electric dipole transitions are between $\mathrm{B}_{1}$ and $\mathrm{B}_{2}$ levels of identical statistical weights, meaning (Table 5) that $a$-type transitions occur within a given tunnelling state, while those of $b$-type take place between tunnelling levels. Clearly, the tunnelling effect essentially modulates the $b$ component of the dipole moment.

Spectral lines falling in the $170-500 \mathrm{GHz}$ frequency range have been predicted using the computed energy difference between the rotationless $(N=0)$ levels of the two tunnelling substates $\Delta E_{01} \approx 30 \mathrm{MHz}$ (Nesbitt et al. 2014) together with the ab initio molecular parameters given in Tables 2-4 except for the $B$ and $C$ ground vibrational state rotational constants, which were obtained by fitting reported IR lines of the $v_{3} \mathrm{CH}$ symmetric stretching band (Roberts et al. 2013). These correspond to transitions from the lower tunnelling state $(v=0)$. All groundstate molecular parameters were taken to be identical in the two tunnelling states.

\section{Experimental details}

\subsection{Spectrometer}

Millimeter-wave (mmw) $b$-type transitions belonging to $Q$ and $R$ branches were recorded using the Lille spectrometer, which 
is devoted to the study of short-lived species. We failed to detect rotational-tunnelling transitions of $a$-type. The spectrometer was adapted for the production of the $\mathrm{CH}_{2} \mathrm{OH}$ radical, and the salient features are summarised here. It now employs an all-solid-state frequency multiplier chain for emission between $170-500 \mathrm{GHz}$. Mmw radiation was obtained by the frequency multiplication of an E8257D synthesizer with option UNX for improved phase noise performance (Agilent, 8.88-13.89 GHz). It was referenced to a GPS-locked Rubidium atomic clock (FS725, Stanford Research Systems) to secure its frequency accuracy. The output of the synthesizer fed a WR9.0 Amplifier Multiplier Chain (Virginia Diodes Inc.) capable of delivering an output power up to $14 \mathrm{dBm}$ in the $80-125 \mathrm{GHz}$ frequency band. It was used to drive two active broadband frequency doublers (models WR4.3 and WR2.2) and one passive full-band tripler (WR2.8) to cover the $170-500 \mathrm{GHz}$ region entirely. For improved sensitivity, the synthesizer was frequency-modulated at $27 \mathrm{kHz}$, and phasesensitive detection at twice this frequency was accomplished with a lock-in amplifier (SR830, Stanford Research Systems), resulting in second-derivative line shapes. The fine-tuning of the modulation depth to the line widths and line strengths ensured full optimization of signal detection, which was achieved using a fast liquid-helium-cooled InSb hot-electron bolometer (QMC Instruments Ltd). This multiplier chain was controlled by a custom-made data acquisition program running in the Igor Pro environment (Wavemetrics) also employed for signal processing. The E8257D synthesizer was either digitally swept step-bystep for spectral search or analogically fast-scanned upwards and downwards. This latter mode of operation, in combination with a 12-bit resolution HDO4034 oscilloscope (Teledyne Lecroy) equipped with a low-noise system architecture, is especially advantageous to efficiently accumulate weak spectra. Accuracy of the measurements was checked against known lines of formaldehyde, and is estimated to be in the $50-150 \mathrm{kHz}$ range depending on the signal-to-noise $(\mathrm{S} / \mathrm{N})$ ratio obtained for each line.

\subsection{Production of $\mathrm{CH}_{2} \mathrm{OH}$}

The $\mathrm{CH}_{2} \mathrm{OH}$ radical was generated in a fast flow system by hydrogen abstraction from $\mathrm{CH}_{3} \mathrm{OH}$ by $\mathrm{Cl}$. The single-pass absorption cell consisted of a $2 \mathrm{~m}$ long double jacketed Pyrex tubing to cool the gas mixture by flowing liquid-nitrogen between the two jackets. Off-axis parabolic mirrors were placed at either end of the cell to optimize the injection of mmw radiation into the cell and to focus the beam onto the bolometer. It was equipped with a solenoid coil used for creating an axial magnetic field to distinguish paramagnetic lines through the observation of the Zeeman effect by successive on/off measurements. The stationary component of the Earth's magnetic field was not compensated for by the use of Helmholtz coils. At the one end (close to the mmw source), a horizontal Pyrex crosspiece ( $25 \mathrm{~cm}$ long, $20 \mathrm{~cm}$ wide) was connected to introduce upstream methanol vapour and chlorine separately (the two inlets were facing each other). $\mathrm{Cl}_{2}$ diluted with $\mathrm{He}(3.8 \%)$ was used as a source for atomic chlorine. The cylinder containing the $\mathrm{He} / \mathrm{Cl}_{2}$ gas mixture was agitated on a daily basis to keep the gaseous mixture homogeneous. $\mathrm{Cl}$ atoms were generated in a microwave discharge in a small quartz tube (16 cm long, $9 \mathrm{~mm}$ inner diameter) placed at one inlet and surrounded by an Evenson-type cavity fed by radiation from a magnetron operating at $2450 \mathrm{MHz}$. The power of the microwave discharge was kept to the minimum $(20 \mathrm{~W})$. Inlet connections to the Pyrex element were made using Teflon flanges to avoid metal in the chemical reaction zone. At the other end, a vertical Pyrex crosspiece was used to connect to a half-choked diffusion pump below the cell and to a pressure gauge above the cell. The outer end of each crosspiece was sealed by a Teflon window mounted at a Brewster angle to improve transmission of polarized mmw radiation and to reduce standing waves. The optimized partial pressure for methanol was $1.2 \times 10^{-3} \mathrm{mBar}$ and the total pressure measured downstream was $7.0 \times 10^{-3} \mathrm{mBar}$. A brownish deposit located on the inner wall surfaces of the horizontal crosspiece formed rapidly after a few hours of experimentation. Once it began to darken, the detection of $\mathrm{CH}_{2} \mathrm{OH}$ became impossible and the periodic elimination of this deposit was ineluctable. Cooling the $2 \mathrm{~m}$ long tubing element, although not absolutely necessary, was very helpful to efficiently trap the many diamagnetic species generated and otherwise detected, such as unreacted methanol, $\mathrm{H}_{2} \mathrm{CO}, \mathrm{H}_{2} \mathrm{O}, \mathrm{HClCO}, \mathrm{CO}$, and $\mathrm{HCOOH}$. Several unidentified diamagnetic lines were also detected. The lifetime of $\mathrm{CH}_{2} \mathrm{OH}$ in our experimental setup was short enough that the magnetic field created by the solenoid coil, which did not surround the horizontal crosspiece, was inefficient in disturbing the energy levels of the $\mathrm{CH}_{2} \mathrm{OH}$ radical by the Zeeman interaction. Instead, it was appropriate to place strong permanent magnets near the horizontal crosspiece to confirm that the lines belong to this paramagnetic species. Lines of HCO (formyl radical, Blake et al. 1984), which we also observed during our spectral search, needed an additional magnetic field generated by the solenoid coil ( $200 \mathrm{G})$ to completely disappear. The observed weakness of the lines of the hydroxymethyl radical can presumably be imputed to its short lifetime, which is partly due to the fast reaction of $\mathrm{CH}_{2} \mathrm{OH}$ with $\mathrm{Cl}$ to yield $\mathrm{H}_{2} \mathrm{CO}$ and $\mathrm{HCl}$, about one order of magnitude faster than the reaction of $\mathrm{CH}_{3} \mathrm{OH}$ with $\mathrm{Cl}$ (Aristov et al. 2000; Pagsberg et al. 1988), and to the distribution of the radical population between the tunnelling, fine and hyperfine sublevels.

\subsection{Identification of the spectrum}

In the very early stage of the experiment, aiming at testing the chemical reactions, we found it impossible to observe the $N, J=3,3.5 \leftarrow 2,2.5, K=1, F=5 \leftarrow 4$ upper component transition of $\mathrm{CH}_{3} \mathrm{O}$ in the ${ }^{2} E_{3 / 2}$ state $(192603.84 \mathrm{MHz})$. It is known that $\mathrm{Cl}$ atoms exclusively attack one of the three methyl protons in methanol (Dóbé et al. 1994). Formaldehyde was easily detected, and the optimized partial pressure of methanol is slightly below the maximum of formaldehyde detected (observing the $J_{K_{a}, K_{c}}=3_{1,2} \leftarrow 2_{1,1}$ line near $225697.8 \mathrm{MHz}$ ). We then searched for the $6_{15} \leftarrow 6_{06}$ and $7_{16} \leftarrow 7_{07}$ transitions, which were predicted to lie around 209.45 and $225.65 \mathrm{GHz}$. Lines corresponding to the various tunnelling and fine-structure sublevels were expected to appear within a few hundred $\mathrm{MHz}$ around these values. We continuously scanned the $208-228.0 \mathrm{GHz}$ frequency region. The only paramagnetic line detected lies near $227.330 \mathrm{GHz}(\sim 1.9 \mathrm{GHz}$ above the predicted frequency for the $7_{16} \leftarrow 7_{07}$ transition) and appears as a doublet whose components are separated by $\sim 2.3 \mathrm{MHz}$ and have imbalanced intensities. We were not able to detect any other line sensitive to the Zeeman effect within $\pm 700 \mathrm{MHz}$. We afterwards decided to scan between $243.5-247.5 \mathrm{GHz}$ to search for the $8_{17} \leftarrow 8_{08}$ transition, which was predicted to occur at $245.04 \mathrm{GHz}$. We only found one paramagnetic line that showed itself as a triplet near 247.242 GHz. Again we failed to observe another fine-structure component of any tunnelling levels. However, we were convinced that they were due to the $\mathrm{CH}_{2} \mathrm{OH}$ radical because of the comparable frequency offset of $\sim 2 \mathrm{GHz}$ compared with predictions, and predominantly because of the hyperfine structure and of the chemical conditions for them to be discovered. The search 
for the $9_{18} \leftarrow 9_{09}$ transition proved to be decisive in the identification of the rotational spectrum of $\mathrm{CH}_{2} \mathrm{OH}$. Another doublet was found at $270.105 \mathrm{GHz}, \sim 2.2 \mathrm{GHz}$ above the predicted frequency $(267.87 \mathrm{GHz})$. Scaling the frequency offsets with $N$ led us to conclude that the triplet seen at $247.242 \mathrm{GHz}$ corresponds to the $J=N+S$ fine-structure component of the $v=1 \leftarrow 0$ level, while the doublets observed at $227.330 \mathrm{GHz}$ and $270.105 \mathrm{GHz}$ are the $J=N+S$ transitions arising from the $v=1$ tunnelling level. The averaged frequencies of the hyperfine components were least-squares analysed in a preliminary fit with the reported IR lines of the $v_{3}$ band (Roberts et al. 2013), which allowed us to predict and detect within several $\mathrm{MHz}$ the remaining fine-structure components of the $9_{18} \leftarrow 9_{09}$ transition (arising from the two tunnelling levels), except for the one occurring near $270.530 \mathrm{GHz}$ that corresponds to $J=N-S, v=0 \leftarrow 1$, which overlaps with unidentified diamagnetic lines. The fine-structure components of the $7_{16} \leftarrow 7_{07}$ and $8_{17} \leftarrow 8_{08}$ transitions that we initially missed are either too weak or masked by lines of diamagnetic species. The triplet detected at $247.242 \mathrm{GHz}$ is composed of six hyperfine components, and only four of them could be discovered in a single scan (two of which are blended, plus two adjacent lines).

\section{Spectral analysis}

With a doublet electronic ground state $(S=1 / 2)$, each tunnelling level for $N>0$ exhibits a fine structure of rotational $J$ sublevels with $J=N \pm 1 / 2$. For $N=0, J=1 / 2$ is the only possible spin sublevel. The electron spin-rotation coupling tensor, which contains five non-zero elements (the two off-diagonal terms $\epsilon_{a b}$ and $\epsilon_{b a}$ in addition to the three diagonal $\epsilon_{i i}$, Brown \& Sears 1979) causes the fine structure. From the Pauli exclusion principle it follows (Table 5) that levels with a statistical weight of 3(1) are associated with $I_{\mathrm{H}}=1(0)$ and correspond to the ortho-(para-) isomer of the $\mathrm{CH}_{2} \mathrm{OH}$ radical. Even and odd values of $K_{a}$ in the $v=0$ and 1 tunnelling levels, respectively, connect with the ortho species (Table 5). Consequently, the above-mentioned finestructure levels of para- $\mathrm{CH}_{2} \mathrm{OH}$ further split into two hyperfine sublevels that are due solely to the hydroxyl hydrogen nucleus, while those of the ortho isomer split into six hyperfine sublevels owing to the additional two equivalent methylenic proton spins. Figures 2 and 3 illustrate such a hyperfine pattern alternation observed in the para- and ortho-isomer of the hydroxymethyl radical, respectively.

We employed the following coupling scheme of angular momenta: $\boldsymbol{J}=\boldsymbol{N}+\boldsymbol{S}, \boldsymbol{F}_{1}=\boldsymbol{J}+\boldsymbol{I}_{\mathrm{H}_{1}}$ for para- $\mathrm{CH}_{2} \mathrm{OH}$, and $\boldsymbol{J}=\boldsymbol{N}+\boldsymbol{S}$, $\boldsymbol{F}_{1}=\boldsymbol{J}+\boldsymbol{I}_{\mathrm{H}_{1}}, \boldsymbol{I}_{\mathrm{H}}=\boldsymbol{I}_{\mathrm{H}_{2}}+\boldsymbol{I}_{\mathrm{H}_{3}}$ and $\boldsymbol{F}=\boldsymbol{F}_{1}+\boldsymbol{I}_{\mathrm{H}}$ for the ortho isomer. Then each rotational-tunnelling level is identified with $N, K_{a}, K_{c}, J, F_{1}, F$, and $v$ as quantum numbers, the latter one describing the tunnelling substate. The Hamiltonian used in the analysis is that for an asymmetric top radical in a doublet state including the energy separation between the tunnelling levels, that is to say, no interaction terms connecting the two tunnelling substates were needed to account for the observed spectrum: $H=H_{\text {rot }}+H_{\text {sr }}+H_{\mathrm{ns}}+H_{01}$. Details on these terms can be found in Tanaka et al. (2004). Briefly, $H_{\text {rot }}$ and $H_{\text {sr }}$ stand for the Watson $A$ reduction in the $\mathrm{I}^{\mathrm{r}}$ representation of the rotational and of the electron-spin rotational coupling tensors, respectively, both including the centrifugal distortion effects. $H_{\mathrm{ns}}$ $\left(=H_{\mathrm{ns}}\left(\mathrm{H}_{1}\right)+H_{\mathrm{ns}}\left(\mathrm{H}_{2}\right)+H_{\mathrm{ns}}\left(\mathrm{H}_{3}\right)\right)$ describe the Fermi contact (isotropic, $a_{F}$ ) and the magnetic dipole-dipole (anisotropic, $\boldsymbol{T}$ ) electron spin-nuclear spin interactions owing to the three hydrogen nuclei contributions. Bowater et al. (1973) have shown for a paramagnetic species of $C_{\mathrm{s}}$ symmetry that the tensors $\boldsymbol{T}$ contain

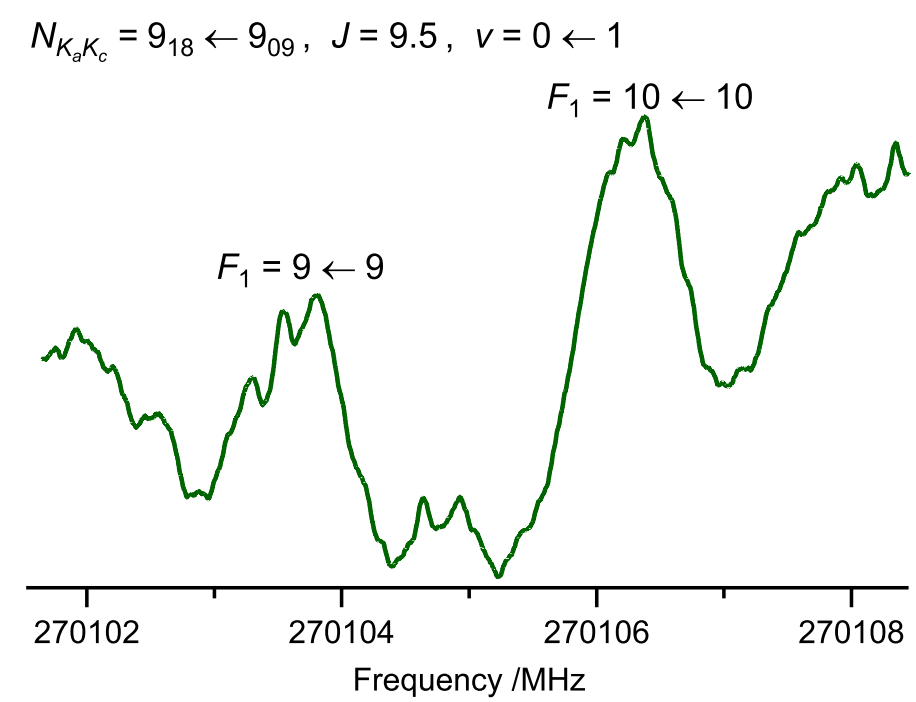

Fig. 2. Hyperfine structure of para- $\mathrm{CH}_{2} \mathrm{OH}$ for the ${ }^{r} Q_{0}(9)$ branch transition with $J=9.5 \leftarrow 9.5$ arising from the lower tunnelling state. The record consists of 40 accumulations acquired at a rate of $1.360 \mathrm{kHz} / \mathrm{s}$ for each direction (e.g. upwards) using a time constant of $30 \mathrm{~ms}$ at the lock-in amplifier.

only one $\left(T_{a b}\right)$ non-zero off-diagonal coupling constants. The observed transitions did not permit us to determine any of the offdiagonal parameters. The last contribution to the Hamiltonian, $H_{01}$, contains $\Delta E_{01}$, the energy difference between the rotationless $(N=0)$ levels of the two tunnelling substates. The observed transitions limited to $\Delta K_{a}= \pm 1$ (i.e. odd $\Delta K_{a}$ ) prevented the Coriolis tunnelling interaction Hamiltonian from being included in the analysis (Schäfer et al. 2003).

In total, $96 \mathrm{mmw}$ lines were recorded between $197-402 \mathrm{GHz}$ and assigned to $b$-type $Q$ - and $R$-branches transitions, involving $N^{\prime \prime}=5-13, K_{a}^{\prime \prime}=0,1$. They were subjected to a global least-squares analysis with available IR lines of the $v_{3}$ band, with the modified version of Pickett's program SPFIT (to circumvent the size limitation of the Hamiltonian) using 21 adjustable parameters (Pickett 1991; Kisiel 2001). The measured frequencies were weighted proportionally to the inverse square of their experimental uncertainties. In the least-squares analysis, the $A$ rotational constant was constrained to be identical in all states (i.e. in the tunnelling levels of the ground- and $v_{3}$ vibrational states). Because of strong correlations between the $C$ rotational constants of the two tunnelling levels (and to a less extent between the two $B$ ), we instead fitted $B$ and $C$ of the lower tunnelling level $(v=0)$ and the differences of those in the upper level $(v=1)$ relatively to the lower level values. The standard deviations of the fit are $77 \mathrm{kHz}$ and $0.09 \times 10^{-3} \mathrm{~cm}^{-1}$ for the mmw and IR transitions, respectively. The ground-state constants derived are provided in Tables 2-4 for comparison with their computed values. Generally speaking, excellent agreement is found between them, and the nearly identical values of the $B$ and $C$ rotational constants in the two tunnelling substates are consistent with a high $\mathrm{OH}$ torsional barrier height and small energy difference between the tunnelling levels, as in the case of the $\mathrm{CHF}_{2}$ radical (Inada et al. 1998). The molecular parameters that we obtained have been used to predict lines of astronomical interest using the MADEX code (Cernicharo 2012), namely the $N_{K_{a} K_{c}}=1_{10}-1_{01}$ and the fundamental one, $1_{11}-0_{00}$, whose upper energy levels lie below $10 \mathrm{~K}$. The most intense of them are supplied in Table 6. A supplementary spectrum to this table is plotted in Fig. 4, which lists expected line intensities for cold 


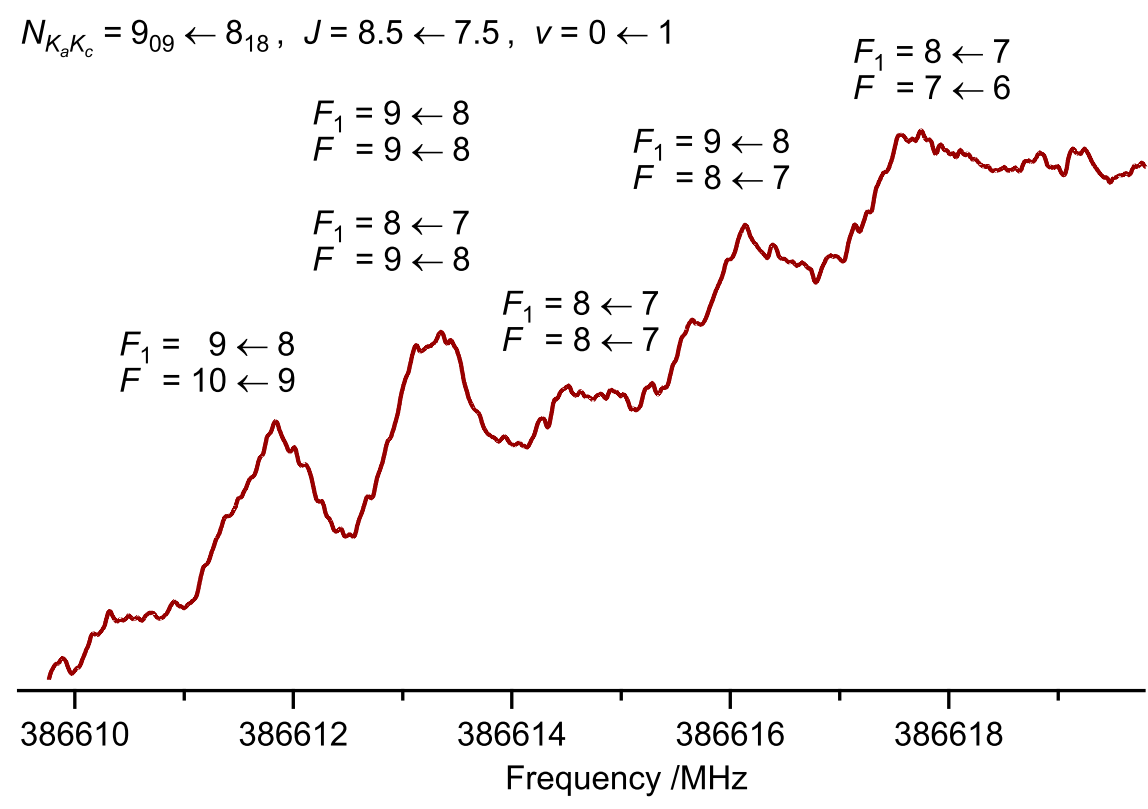

Fig. 3. Hyperfine structure of ortho- $\mathrm{CH}_{2} \mathrm{OH}$ for the ${ }^{p} R_{1}(8)$ branch transition from the lower tunnelling state with $J=8.5 \leftarrow 7.5$.

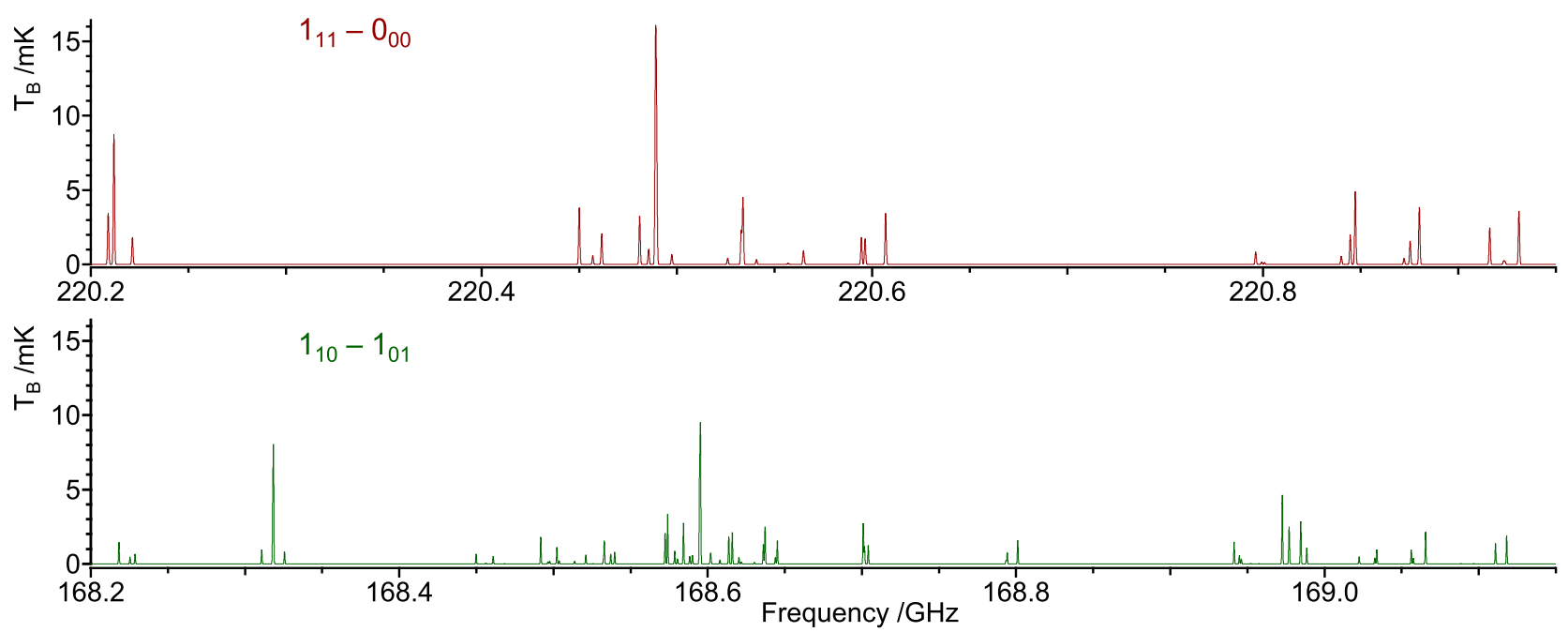

Fig. 4. Expected line intensities for the two strongest series of hyperfine lines for a cold prestellar core with a column density $N\left(\mathrm{CH}_{2} \mathrm{OH}\right)=10^{12} \mathrm{~cm}^{-2}$, full line width at half power $\Delta v=1 \mathrm{~km} \mathrm{~s}^{-1}$, and $T_{\mathrm{K}}=12 \mathrm{~K}$. The predictions have been obtained with the MADEX code (Cernicharo 2012). The upper panel corresponds to the fundamental $R$-branch transition $1_{11}-0_{00}$ around $220.5 \mathrm{GHz}$ and the lower one to the $Q$-branch transition $1_{10}-1_{01}$ near $168.6 \mathrm{GHz}$. Quantum numbers for the selected hyperfine components can be found in Table 6 . We have assumed that the source fills the main beam of the telescope.

prestellar cores around 168.6 and $220.5 \mathrm{GHz}$. The list of $o b s$. and $o b s$. - cal. transition frequencies is supplied in Table A.1.

Finally, the inertial defect has been inferred from the rotational constants, $\Delta_{0}=-0.0406$ amu $\AA^{2}$, a low value compatible with a planar structure. The fact that $\Delta_{0}$ is negative deserves a last comment. Oka (1995) gave a semi-empirical formula relating the zero-point inertial defect, the lowest out-of-plane vibration $v_{l}$ (in unit of $\mathrm{cm}^{-1}$ ), and $\sqrt{I_{c}}$, where $I_{c}$ is the principal moment of inertia along the $c$ axis: $\Delta_{0}=-33.715 / v_{l}+0.0186 \sqrt{I_{c}}$. Hence very low out-of-plane vibration can give a dominant negative contribution to the inertial defect. We obtained $v_{l}=274 \mathrm{~cm}^{-1}$, a value in excellent agreement with the observed one at $234 \pm 5 \mathrm{~cm}^{-1}$ for the $v_{9}-\mathrm{CH}_{2}$ wag mode (Johnson \& Hudgens 1996).

\section{Conclusion}

The rotational-tunnelling spectrum of the $\mathrm{CH}_{2} \mathrm{OH}$ radical has been identified for the first time. Several molecular parameters have been accurately determined, not the least of which is the energy difference between the rotationless levels of the two tunnelling substates $(139.73 \pm 0.10 \mathrm{MHz})$. The height of the $\mathrm{OH}$ torsional barrier potential can in principle be obtained from this tunnelling rate, but this determination is beyond the scope of this study. The observed hyperfine structure alternation with $3: 1$ statistical weights depending on the symmetry and parity of the rotational levels revealed a tunnelling $\mathrm{CH}_{2} \mathrm{OH}$ radical with $\mathrm{B}_{1}$ symmetry in the $C_{2 \mathrm{v}}(\mathrm{M})$ permutation-inversion molecular symmetry group $\left(\mathrm{A}^{\prime \prime}\right.$ symmetry assuming a $C_{\mathrm{s}}$ planar and rigid molecular framework). It is worth mentioning that the works reported by the Nesbitt group (Roberts et al. 2013; Wang et al. 2014 b) were invaluable to us to accomplish this research. Several hyperfine transitions have been predicted with enough accuracy to enable the radioastronomical detection of this species, which is important in astrochemistry. Ongoing efforts are underway to increase the radical production efficiency to allow for measurements of higher $K_{a}$ transitions and/or above $400 \mathrm{GHz}$. 
Table 6. Strongest lines of astrophysical interest predicted for a cloud at $T=10 \mathrm{~K}$.

\begin{tabular}{|c|c|c|c|c|c|c|}
\hline$N_{K_{a} K_{c}}$ & $J$ & $F_{1}$ & $F^{a}$ & $v^{b}$ & $f_{\text {cal. }}{ }^{c}$ & $S_{\mathrm{ij}} \mu_{b}^{2 d}$ \\
\hline \multicolumn{7}{|l|}{$1_{10}-1_{01}$} \\
\hline & $1.5-1.5$ & $1-1$ & & 1 & $168317.98(32)$ & 2.7 \\
\hline & $1.5-1.5$ & $2-2$ & & 1 & $168318.43(32)$ & 5.0 \\
\hline & $1.5-1.5$ & $1-1$ & $2-1$ & 0 & $168573.01(61)$ & 1.4 \\
\hline & $1.5-1.5$ & $2-2$ & $3-2$ & 0 & $168574.66(61)$ & 2.3 \\
\hline & $1.5-1.5$ & $2-2$ & $2-1$ & 0 & $168584.76(50)$ & 1.9 \\
\hline & $1.5-1.5$ & $1-1$ & $2-2$ & 0 & $168594.45(48)$ & 4.4 \\
\hline & $1.5-1.5$ & $2-2$ & $3-3$ & 0 & $168595.00(48)$ & 6.3 \\
\hline & $1.5-1.5$ & $1-1$ & $1-1$ & 0 & $168613.73(55)$ & 1.0 \\
\hline & $1.5-1.5$ & $2-2$ & $2-2$ & 0 & $168616.35(60)$ & 1.5 \\
\hline & $1.5-1.5$ & $2-2$ & $2-3$ & 0 & $168636.69(89)$ & 1.6 \\
\hline & $0.5-0.5$ & $1-1$ & & 1 & 168701.04(107) & 1.9 \\
\hline & $0.5-0.5$ & $1-1$ & $2-2$ & 0 & $168973.75(153)$ & 3.1 \\
\hline & $0.5-0.5$ & $0-0$ & $1-1$ & 0 & $168978.11(154)$ & 1.6 \\
\hline & $0.5-0.5$ & $1-1$ & $2-1$ & 0 & $168983.72(134)$ & 1.8 \\
\hline & $0.5-1.5$ & $1-2$ & $1-2$ & 0 & $169065.54(104)$ & 1.5 \\
\hline & $0.5-1.5$ & $0-1$ & $1-2$ & 0 & $169110.62(107)$ & 0.9 \\
\hline & $0.5-1.5$ & $1-2$ & $2-3$ & 0 & $169117.85(107)$ & 1.3 \\
\hline \multicolumn{7}{|l|}{$1_{11}-0_{00}$} \\
\hline & $1.5-0.5$ & $1-0$ & & 1 & $220207.17(32)$ & 1.8 \\
\hline & $1.5-0.5$ & $2-1$ & & 1 & $220210.10(32)$ & 4.5 \\
\hline & $1.5-0.5$ & $1-1$ & & 1 & $220219.57(32)$ & 0.9 \\
\hline & $1.5-0.5$ & $2-1$ & $2-1$ & 0 & $220448.96(79)$ & 2.0 \\
\hline & $1.5-0.5$ & $1-1$ & $1-0$ & 0 & $220460.44(78)$ & 1.0 \\
\hline & $1.5-0.5$ & $2-1$ & $1-1$ & 0 & $220479.62(48)$ & 1.7 \\
\hline & $1.5-0.5$ & $2-1$ & $3-2$ & 0 & $220487.26(36)$ & 6.3 \\
\hline & $1.5-0.5$ & $1-0$ & $2-1$ & 0 & $220487.81(36)$ & 4.1 \\
\hline & $1.5-0.5$ & $1-0$ & $1-1$ & 0 & $220530.92(70)$ & 1.2 \\
\hline & $1.5-0.5$ & $2-1$ & $2-2$ & 0 & $220531.84(69)$ & 2.3 \\
\hline & $0.5-0.5$ & $1-0$ & & 1 & $220592.90(90)$ & 0.9 \\
\hline & $0.5-0.5$ & $0-1$ & & 1 & $220594.79(90)$ & 0.9 \\
\hline & $0.5-0.5$ & $1-1$ & & 1 & $220605.30(90)$ & 1.8 \\
\hline & $0.5-0.5$ & $0-1$ & $1-0$ & 0 & $220843.95(136)$ & 1.0 \\
\hline & $0.5-0.5$ & $1-1$ & $2-1$ & 0 & $220846.48(134)$ & 2.5 \\
\hline & $0.5-0.5$ & $1-0$ & $0-1$ & 0 & $220872.95(117)$ & 0.8 \\
\hline & $0.5-0.5$ & $1-1$ & $1-2$ & 0 & $220877.65(117)$ & 2.0 \\
\hline & $0.5-0.5$ & $0-0$ & $1-1$ & 0 & $220914.42(87)$ & 1.3 \\
\hline & $0.5-0.5$ & $1-1$ & $2-2$ & 0 & 220929.36(87) & 1.8 \\
\hline
\end{tabular}

Notes. ${ }^{(a)}$ For para- $\mathrm{CH}_{2} \mathrm{OH}$, the $F$ quantum number does not exist. (b) The lower tunnelling sublevel is given. ${ }^{(c)}$ Calculated frequencies $(\mathrm{MHz})$ using constants from Tables 2-4. $1 \sigma$ error between parentheses applies to the last digits. ${ }^{(d)}$ Line strengths calculated using $\mu_{b}=1.64 \mathrm{D}$.

If successful, these observations will help refine the molecular constants and hence the prediction of lines of astrophysical interest. A direct measure of the $\mathrm{CH}_{2} \mathrm{OH}$ radical abundance will be of high relevance for the modelling of the rich chemistry that results in the formation of complex organic molecules in cold dark cores, such as B1-b and TMC1, and also in hot cores and corinos, where dust ice mantles are submitted to strong desorption processes. A first check in Orion using IRAM $30 \mathrm{~m}$ observations and ALMA Science Verification data provided an upper limit to the column density of $\mathrm{CH}_{2} \mathrm{OH}$ of $1 / 1000$ that of methanol. In cold dark clouds the higher partition function and lower dipole moment than $\mathrm{CH}_{3} \mathrm{O}$ will translate into lines weaker than those of methoxy by a factor of 3-4. These results will be published in a forthcoming paper.

Acknowledgements. This research was supported in part by the National French Program "Physique et Chimie du Milieu Interstellaire" (PCMI) and the French National Research Agency (ANR-13-BS05-0008-02 "IMOLABS"). The Laboratoire d'Excellence "Chemical and Physical Properties of the Atmosphere" (CaPPA) is also acknowledged. The CaPPA project is funded by the French National Research Agency under contract ANR-11-LABX-0005-01, by the Regional Council "Hauts de France" and by the European Regional Development Fund (ERDF). J. Cernicharo thanks the European Research Council and the Spanish Ministry of Science and Innovation through the NANOCOSMOS (SyG-610256) and ASTROMOL (CSD2009-00038) projects, respectively, and the Spanish Ministry of Economy and Competitiveness through the projects AYA2009-07304, and AYA2012-32032. The referee is also thanked for the valuable comments concerning the hyperfine structure.

\section{References}

Aristov, V., Conroy, D., \& Reisler, H. 2000, Chem. Phys. Lett., 318, 393 Atkinson, R. 1990, Atmos. Environ. Part A, 24, 1

Blake, G. A., Sastry, K. V. L. N., \& de Lucia, F. C. 1984, J. Chem. Phys., 80, 95 Bowater, I. C., Brown, J. M., \& Carrington, A. 1973, Proc. Roy. Soc. London A, 333,265

Brown, J. M., \& Sears, T. J. 1979, J. Mol. Spectrosc., 75, 111

Bruna, P. J., \& Grein, F. 1998, J. Phys. Chem. A, 102, 3141

Bunker, P. R., \& Jensen, P. 1998, Molecular Symmetry and Spectroscopy, 2nd edn. (Cambridge: NRC Researcch Press)

Cernicharo, J. 2012, in ECLA-2011: Proc. European Conference on Laboratory Astrophysics, eds. C. Stehl, C. Joblin, \& L. d'Hendecourt, EAS Pub. Ser., 58, 251

Cernicharo, J., Marcelino, N., Roueff, E., et al. 2012, ApJ, 759, L43

Chang, C.-H., Wang, F., \& Nesbitt, D. J. 2014, 69th International Symposium on Molecular Spectroscopy, http://hdl .handle.net/2142/50829

Dóbé, S., Bérces, T., Temps, F., Wagner, H. C., \& Ziemer, H. 1994, 25th Symp. (Int.) Combust. [Proc.], 25, 755

Frisch, M. J., Trucks, G. W., Schlegel, H. B., et al. 2009, Gaussian 09 Revision E.01, Gaussian Inc. Wallingford CT 2009

Gerakines, P. A., Schutte, W. A., \& Ehrenfreund, P. 1996, A\&A, 312, 289

Inada, N., Saito, K., Hayashi, M., Ozeki, H., \& Saito, S. 1998, Chem. Phys. Lett., 284,142

Johnson, R. D., \& Hudgens, J. W. 1996, J. Phys. Chem., 100, 19874

Kamarchik, E., Rodrigo, C., Bowman, J. M., Reisler, H., \& Krylov, A. I. 2012, J. Chem. Phys., 136, 084304

Kisiel, Z. 2001, Spectroscopy from Space, Proc. NATO Advanced Research Workshop held 31 October-4 November 2000, in Bratislava, Slovakia, eds. J. Demaison, K. Sarka, \& E. A. Cohen (Dordrecht: Kluwer Academic Publishers), 91

Lin, J. J., Harich, S., Lee, Y. T., \& Yang, X. 1999, J. Chem. Phys., 110, 10821

Lin, J. J., Shu, J., Lee, Y. T., \& Yang, X. 2000, J. Chem. Phys., 113, 5287

Nesbitt, D. J., Chang, C.-H., \& Wang, F. 2014, 69th International Symp. on Molecular Spectroscopy, http://hdl . handle.net/2142/50831

Nesbitt, F. L., Payne, W. A., \& Stief, L. J. 1989, J. Phys. Chem., 93, 5158

Öberg, J. K., Bottinelli, S., Jörgensen, J. K., \& van Dischoek, E. F. 2010, ApJ, 716,825

Oka, T. 1995, J. Mol. Struct., 352, 225

Pagsberg, P., Munk, J., \& Sillesen, A. 1988, Chem. Phys. Lett., 146, 375

Pagsberg, P., Munk, J., Anastasi, C., \& Simpson, V. J. 1989, J. Phys. Chem., 93, 5162

Pickett, H. M. 1991, J. Mol. Spectrosc., 148, 371

Rasmussen, C. L., Wassard, K. H., Dam-Johansen, K., \& Glarborg, P. 2008, Int. J. Chem. Kinet., 40, 423

Rissanen, M. P., Arppe, S. L., \& Timonen, R. S. 2013, J. Phys. Chem. A, 117, 3902

Roberts, M. A., Sharp-Williams, E. N., \& Nesbitt, D. J. 2013, J. Phys. Chem. A, 117,7042

Schäfer, M., Ha, T.-H., \& Bauder, A. 2003, J. Chem. Phys., 119, 8404

Tanaka, K., Toshimitsu, M., Harada, K., \& Tanaka, T. 2004, J. Chem. Phys., 120, 3604

Wang, F., Chang, C.-H., \& Nesbitt, D. J. 2014a, 69th International Symp. on Molecular Spectroscopy, http://hdl . handle.net/2142/50881

Wang, F., Chang, C.-H., \& Nesbitt, D. J. 2014b, 69th International Symp. on Molecular Spectroscopy, http://hdl . handle.net/2142/50878

Watanabe, N., \& Kouchi, A. 2002, ApJ, 571, L173

Watanabe, N., Mouri, O., Nagaoka, A., et al. 2007, ApJ, 668, 1001 


\section{Appendix A: Additional table}

Table A.1. List of $o b s$. and $o b s$. cal. transition frequencies.

\begin{tabular}{|c|c|c|c|c|c|c|c|c|c|c|c|c|c|}
\hline$N_{K_{a} K_{c}}$ & $J$ & $F_{1}$ & $F^{a}$ & $v^{b}$ & $o b s .^{c}$ & obs.-cal. ${ }^{d}$ & $N_{K_{a} K_{c}}$ & $J$ & $F_{1}$ & $F^{a}$ & $v^{b}$ & $o b s .^{c}$ & obs. - cal. $^{d}$ \\
\hline \multirow{2}{*}{$5_{14}-5_{05}$} & $5.5-5.5$ & $5-5$ & & 1 & $197464.267(70)$ & 0.124 & $11_{110}-11_{011}$ & $11.5-11.5$ & $11-11$ & & & $327765.463(60)$ & 0.000 \\
\hline & $5.5-5.5$ & $6-6$ & & 1 & $197466.208(100)$ & 0.114 & & $11.5-11.5$ & $12-12$ & & 1 & $327768.371(50)$ & 0.058 \\
\hline \multirow[t]{2}{*}{$6_{15}-6_{06}$} & $6.5-6.5$ & $6-6$ & & & $210920.459(60)$ & -0.084 & & $10.5-10.5$ & $11-11$ & & 1 & $328301.285(50)$ & 0.042 \\
\hline & $6.5-6.5$ & $7-7$ & & & $210922.652(60)$ & -0.004 & & $10.5-10.5$ & $10-10$ & & & $328304.694(50)$ & 0.008 \\
\hline \multirow{10}{*}{$7_{16}-7_{07}$} & $7.5-7.5$ & $7-7$ & & & $227328.350(60)$ & -0.077 & & $11.5-11.5$ & $11-11$ & $10-10$ & 0 & $328029.021(80)$ & -0.139 \\
\hline & $7.5-7.5$ & $8-8$ & & & $227330.620(60)$ & -0.076 & & $11.5-11.5$ & $12-12$ & $11-11$ & 0 & $328031.528(100)$ & -0.417 \\
\hline & $6.5-6.5$ & $7-7$ & & & $227659.877(60)$ & 0.096 & & $11.5-11.5$ & $11-11$ & $11-11$ & & $328031.528(100)$ & 0.054 \\
\hline & $6.5-6.5$ & $6-6$ & & & $227662.665(100)-$ & -0.135 & & $11.5-11.5$ & $11-11$ & $12-12$ & 0 & $328032.285(90)$ & -0.104 \\
\hline & $7.5-7.5$ & $7-7$ & $6-6$ & 0 & $227604.358(80)$ & -0.025 & & $11.5-11.5$ & $12-12$ & $12-12$ & 0 & $328034.237(90)$ & -0.020 \\
\hline & $7.5-7.5$ & $8-8$ & $7-7$ & 0 & & & & $11.5-11.5$ & $12-12$ & $13-13$ & 0 & $328035.236(90)$ & 0.003 \\
\hline & $7.5-7.5$ & $7-7$ & $7-7$ & 0 & $227606.141(80)$ & 0.097 & & $10.5-10.5$ & $11-11$ & $12-12$ & 0 & & \\
\hline & $7.5-7.5$ & $7-7$ & $8-8$ & & $227606.141(80)$ & -0.147 & & $10.5-10.5$ & $10-10$ & $11-11$ & 0 & & \\
\hline & $7.5-7.5$ & $8-8$ & $8-8$ & 0 & $227608.306(80)$ & 0.065 & & $10.5-10.5$ & $11-111$ & $11-11$ & 0 & & \\
\hline & $7.5-7.5$ & $8-8$ & $9-9$ & & $227608.306(80)$ & -0.239 & & $10.5-10.5$ & $10-101$ & $10-10$ & 0 & & \\
\hline \multirow{10}{*}{$8_{17}-8_{08}$} & $8.5-8.5$ & $8-8$ & & & $246966.022(60)$ & -0.031 & & $10.5-10.5$ & $11-111$ & $10-10$ & 0 & & \\
\hline & $8.5-8.5$ & $9-9$ & & & $246968.468(60)$ & -0.008 & & $10.5-10.5$ & $10-10$ & $9-9$ & 0 & $328572.325(60)$ & 0.013 \\
\hline & $7.5-7.5$ & $8-8$ & & & $247341.946(60)$ & $0.715 *$ & $12_{111}-12_{012}$ & $12.5-12.5$ & $12-12$ & & 1 & $362557.079(60)$ & -0.055 \\
\hline & $7.5-7.5$ & $7-7$ & & & $247345.254(60)$ & $0.906 *$ & & $12.5-12.5$ & $13-13$ & & & $362560.063(80)$ & -0.037 \\
\hline & $8.5-8.5$ & $8-8$ & $7-7$ & 0 & $247239.960(60)$ & 0.045 & & $11.5-11.5$ & $12-12$ & & 1 & $363151.666(60)-$ & -0.039 \\
\hline & $8.5-8.5$ & $9-9$ & $8-8$ & & $247242.263(60)$ & -0.020 & & $11.5-11.5$ & $11-11$ & & 1 & $363155.152(60)-$ & -0.092 \\
\hline & $8.5-8.5$ & $8-8$ & $9-9$ & 0 & $247242.263(60)$ & -0.024 & & $12.5-12.5$ & $13-131$ & $13-13$ & 0 & $362820.961(120)-$ & -0.138 \\
\hline & $8.5-8.5$ & $8-8$ & $8-8$ & 0 & 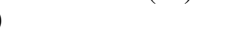 & & & $12.5-12.5$ & $13-131$ & $14-14$ & 0 & $362822.137(80)$ & 0.041 \\
\hline & $8.5-8.5$ & $9-9$ & $9-9$ & 0 & $247244.234(60)$ & 0.110 & & $11.5-11.5$ & $12-121$ & $13-13$ & 0 & $363406.389(80)$ & 0.025 \\
\hline & $8.5-8.5$ & $9-9$ & $10-10$ & 0 & $247244.668(60)$ & -0.032 & & $11.5-11.5$ & $11-111$ & $12-12$ & 0 & $363409.756(80)$ & -0.077 \\
\hline \multirow{16}{*}{$9_{18}-9_{09}$} & $9.5-9.5$ & $9-9$ & & & $270103.657(60)$ & 0.003 & & $11.5-11.5$ & $12-121$ & $12-12$ & 0 & $363409.756(80)$ & 0.254 \\
\hline & $9.5-9.5$ & $10-10$ & & 1 & $270106.240(60)$ & 0.012 & & $11.5-11.5$ & $11-111$ & $11-11$ & 0 & $363413.087(80)$ & 0.128 \\
\hline & $8.5-8.5$ & $9-9$ & & 1 & & & & $11.5-11.5$ & $12-121$ & $11-11$ & 0 & $363414.369(80)$ & -0.014 \\
\hline & $8.5-8.5$ & $8-8$ & & 1 & & & & $11.5-11.5$ & $11-111$ & $10-10$ & 0 & $363417.898(80)$ & -0.016 \\
\hline & $9.5-9.5$ & $9-9$ & $8-8$ & & $270374.935(100)$ & 0.076 & $13_{112}-13_{013}$ & $13.5-13.5$ & $13-13$ & & 1 & & \\
\hline & $9.5-9.5$ & $10-10$ & $9-9$ & & $270377.474(90)$ & $0.099 *$ & & $13.5-13.5$ & $14-14$ & & 1 & $401343.878(120)$ & 0.080 \\
\hline & $9.5-9.5$ & $9-9$ & $9-9$ & & $270377.474(90)$ & $0.590 *$ & & $12.5-12.5$ & $13-13$ & & 1 & $401994.391(120)$ & 0.129 \\
\hline & $9.5-9.5$ & $9-9$ & $10-10$ & 0 & $270377.474(90)$ & $-0.122 *$ & & $12.5-12.5$ & $12-12$ & & 1 & & \\
\hline & $9.5-9.5$ & $10-10$ & $10-10$ & 0 & & & $8_{08}-7_{17}$ & $8.5-7.5$ & $8-7$ & $7-6$ & 1 & $321683.266(80)$ & 0.048 \\
\hline & $9.5-9.5$ & $10-10$ & $11-11$ & 0 & & & & $8.5-7.5$ & $8-7$ & $9-8$ & 1 & $321684.490(80)$ & -0.182 \\
\hline & $8.5-8.5$ & $9-9$ & $10-10$ & 0 & $270795.456(80)$ & $-0.484 *$ & & $8.5-7.5$ & $8-7$ & $8-7$ & 1 & $321684.490(80)$ & 0.070 \\
\hline & $8.5-8.5$ & $8-8$ & $9-9$ & & $270798.448(80)$ & $-0.656 *$ & & $8.5-7.5$ & $9-8$ & $8-7$ & 1 & $321684.490(80)$ & 0.123 \\
\hline & $8.5-8.5$ & $9-9$ & $9-9$ & & $270798.448(80)$ & $-0.314 *$ & & $8.5-7.5$ & $9-8$ & $10-9$ & 1 & $321685.704(80)$ & -0.262 \\
\hline & $8.5-8.5$ & $8-8$ & $8-8$ & & $270801.464(80)$ & $-0.446 *$ & & $8.5-7.5$ & $9-8$ & $9-8$ & 1 & $321685.704(80)$ & 0.120 \\
\hline & $8.5-8.5$ & $9-9$ & $8-8$ & & $270802.655(80)$ & $-0.347 *$ & & $7.5-6.5$ & $8-7$ & $9-8$ & 1 & $321831.092(120)$ & 0.014 \\
\hline & $8.5-8.5$ & $8-8$ & $7-7$ & & $270805.852(80)$ & $-0.360 *$ & & $7.5-6.5$ & $8-7$ & $8-7$ & & $321832.757(120)$ & 0.046 \\
\hline \multirow{16}{*}{$10_{19}-10_{010}$} & $10.5-10.5$ & $10-10$ & & & $296978.021(60)$ & 0.003 & & $7.5-6.5$ & $7-6$ & $8-7$ & 1 & $321832.757(120)$ & 0.165 \\
\hline & $10.5-10.5$ & $11-11$ & & & $296980.826(60)$ & 0.090 & & $7.5-6.5$ & $7-6$ & $7-6$ & 1 & $321834.312(120)$ & 0.140 \\
\hline & $9.5-9.5$ & $10-10$ & & & $297456.630(65)$ & 0.007 & & $7.5-6.5$ & $8-7$ & $7-6$ & 1 & $321835.778(120)-$ & -0.037 \\
\hline & $9.5-9.5$ & $9-9$ & & & $297459.944(65)$ & -0.016 & & $7.5-6.5$ & $7-6$ & $6-5$ & & $321837.260(120)-$ & -0.180 \\
\hline & $10.5-10.5$ & $10-10$ & $9-9$ & 0 & $297245.907(60)$ & 0.036 & $9_{09}-8_{18}$ & $9.5-8.5$ & $9-8$ & $8-7$ & 1 & & \\
\hline & $10.5-10.5$ & $10-10$ & $10-10$ & & $297248.203(90)$ & 0.151 & & $9.5-8.5$ & $9-8$ & $10-9$ & 1 & $386458.220(80)-$ & -0.043 \\
\hline & $10.5-10.5$ & $11-11$ & $10-10$ & & $297248.841(90)$ & 0.312 & & $9.5-8.5$ & $9-8$ & $9-8$ & 1 & $386458.220(80)-$ & -0.029 \\
\hline & $10.5-10.5$ & $10-10$ & $11-11$ & & $297248.841(90)$ & -0.053 & & $9.5-8.5$ & $10-9$ & $9-8$ & 1 & $386458.220(80)-$ & -0.034 \\
\hline & $10.5-10.5$ & $11-11$ & $11-11$ & & $297250.625(80)$ & -0.079 & & $9.5-8.5$ & $10-9 \quad 1$ & $11-10$ & 1 & $386459.395(60)-$ & -0.033 \\
\hline & $10.5-10.5$ & $11-11$ & $12-12$ & 0 & $297251.685(70)$ & 0.080 & & $9.5-8.5$ & $10-9$ & $10-9$ & 1 & $386459.395(60)$ & 0.101 \\
\hline & $9.5-9.5$ & $10-10$ & $11-11$ & 0 & & & & $8.5-7.5$ & $9-8$ & $10-9$ & & $386611.859(60)-$ & -0.028 \\
\hline & $9.5-9.5$ & $9-9$ & $10-10$ & & $297723.876(60)$ & -0.265 & & $8.5-7.5$ & $9-8$ & $9-8$ & 1 & $386613.207(60)-$ & -0.034 \\
\hline & $9.5-9.5$ & $10-10$ & $10-10$ & 0 & $297723.876(60)$ & 0.064 & & $8.5-7.5$ & $8-7$ & $9-8$ & 1 & $386613.207(60)-$ & -0.011 \\
\hline & $9.5-9.5$ & $9-9$ & $9-9$ & 0 & $297727.035(60)$ & -0.038 & & $8.5-7.5$ & $8-7$ & $8-7$ & 1 & & \\
\hline & $9.5-9.5$ & $10-10$ & $9-9$ & 0 & $297728.193(150)$ & -0.050 & & $8.5-7.5$ & $9-8$ & $8-7$ & 1 & $386616.182(60)$ & 0.025 \\
\hline & $9.5-9.5$ & $9-9$ & $8-8$ & 0 & $297731.646(150)$ & 0.079 & & $8.5-7.5$ & $8-7$ & $7-6$ & 1 & $386617.609(60)$ & 0.012 \\
\hline
\end{tabular}

Notes. ${ }^{(a)}$ For para- $\mathrm{CH}_{2} \mathrm{OH}$, the $F$ quantum number does not exist. ${ }^{(b)}$ The lower tunnelling state is given. ${ }^{(c)}$ Observed frequencies (in MHz) with their estimated error measurement given in parentheses applies to the last digits. Unresolved (blended) transitions were fitted with their averaged frequencies. ${ }^{(d)}$ Lines with an (obs. - cal.) value higher than 1.9 times their error measurement were rejected from the fit and are marked with a $*$. 